\title{
Origins Space Telescope science drivers to design traceability
}

\author{
Margaret Meixner, ${ }^{\text {a,b,c, } * \text { Asantha Cooray, }}{ }^{\mathrm{d}}$ David T. Leisawitz ${ }^{\mathrm{e}}{ }^{\mathrm{e}}$ \\ Johannes G. Staguhn $\odot,{ }^{\text {cee }}$ Lee Armus, ${ }^{\text {f }}$ Cara Battersby $\odot,{ }^{\text {g }}$ James Bauer, \\ Dominic Benford, ${ }^{i}$ Edwin Bergin, ${ }^{j}$ Charles Matt Bradford, ${ }^{k}$ \\ Denis Burgarella $\odot$, Sean Carey, ${ }^{\mathrm{f}}$ Elvire De Beck $\odot{ }^{\mathrm{m}}$ \\ Kimberly Ennico-Smith $\odot,{ }^{\text {n }}$ Jonathan J. Fortney, ${ }^{\circ}$ Maryvonne Gerin $\odot,{ }^{p}$ \\ Frank P. Helmich $\odot{ }^{\mathrm{q}}{ }^{\text {Tiffany Kataria, }}{ }^{\mathrm{k}}$ Eric E. Mamajek $\odot{ }^{\mathrm{k}}$ \\ Gary J. Melnick, ${ }^{r}$ Stefanie N. Milam $\odot{ }^{\text {ec }}$ Samuel Harvey Moseley, \\ Desika Narayanan, ${ }^{\text {s }}$ Susan G. Neff, ${ }^{\mathrm{e}}$ Deborah Padgett, ${ }^{\mathrm{k}}$ \\ Klaus Pontoppidan $\odot$, Alexandra Pope $\odot$, Thomas L. Roellig, \\ Itsuki Sakon $\odot,{ }^{\mathrm{u}}$ Karin Sandstrom $\odot,{ }^{\mathrm{v}}$ Douglas Scott $\odot,{ }^{\mathrm{w}}$ Kartik Sheth, ${ }^{\mathrm{i}}$ \\ Kevin B. Stevenson $\odot,{ }^{b, x}$ Kate Y. Su $\odot,{ }^{y}$ Joaquin Vieira, ${ }^{z}$ \\ Martina C. Wiedner $\odot$, Edward Wright, ${ }^{\text {aa }}$ Jonas Zmuidzinas, ${ }^{\text {ab }}$ \\ and Origins Study Team ${ }^{\dagger}$ \\ ${ }^{a}$ SOFIA Science Mission Operations, NASA Ames Research Center, Moffett Field, \\ California, United States \\ ${ }^{\mathrm{b}}$ Space Telescope Science Institute, Baltimore, Maryland, United States \\ ${ }^{\mathrm{c} J o h n s}$ Hopkins University, Baltimore, Maryland, United States \\ ${ }^{\mathrm{d}}$ University of California, Irvine, Department of Physics and Astronomy, Irvine, \\ California, United States \\ ${ }^{e}$ NASA Goddard Space Flight Center, Greenbelt, Maryland, United States \\ ${ }^{\mathrm{f}}$ Caltech/Infrared Processing and Analysis Center, Pasadena, California, United States \\ ${ }^{\mathrm{g}}$ University of Connecticut, Department of Physics, Storrs, Connecticut, United States \\ ${ }^{h}$ University of Maryland, Astronomy Department, College Park, Maryland, United States \\ iScience Mission Directorate, NASA Headquarters, Astrophysics Division, Washington, \\ DC, United States \\ ${ }^{j}$ University of Michigan, Department of Astronomy, Ann Arbor, Michigan, United States \\ ${ }^{\mathrm{k}}$ Caltech/Jet Propulsion Laboratory, Pasadena, California, United States \\ ${ }^{1}$ Laboratoire d'Astrophysique de Marseille, Marseille, France \\ ${ }^{\mathrm{m}}$ Chalmers Institute of Technology, Department of Space, Earth and Environment, \\ Gothenburg, Sweden \\ ${ }^{n}$ NASA Ames Research Center, Mountain View, California, United States \\ ${ }^{\circ}$ University of California, Santa Cruz, Astronomy and Astrophysics Department, Santa Cruz, \\ California, United States \\ PSorbonne Université, Observatoire de Paris, CNRS, LERMA, Paris, France \\ ${ }^{\mathrm{q}}$ Netherlands Institute for Space Research (SRON), Groningen, The Netherlands \\ ${ }^{r}$ Harvard-Smithsonian Center for Astrophysics, Cambridge, Massachusetts, United States \\ ${ }^{\mathrm{s}}$ University of Florida, Department of Astronomy, Gainesville, Florida, United States \\ tUniversity of Massachusetts, Amherst, Department of Astronomy, Massachusetts, \\ United States \\ "The University of Tokyo, School of Science, Tokyo, Japan \\ ${ }^{v}$ CASS, University of California, San Diego, San Diego, California, United States \\ ${ }^{\text {w} U n i v e r s i t y ~ o f ~ B r i t i s h ~ C o l u m b i a, ~ D e p a r t m e n t ~ o f ~ P h y s i c s ~ a n d ~ A s t r o n o m y, ~ V a n c o u v e r, ~}$ \\ British Columbia, Canada \\ ${ }^{\mathrm{x}}$ Johns Hopkins Applied Physics Lab, Laurel, Maryland, United States \\ ${ }^{\mathrm{y}}$ The University of Arizona, Department of Astronomy and Steward Observatory, Tucson, \\ Arizona, United States \\ ${ }^{\mathrm{z}}$ University of Illinois, Department of Astronomy, Urbana-Champaign, Urbana, Illinois, \\ United States \\ ${ }^{\text {aa } U C L A, ~ D i v i s i o n ~ o f ~ A s t r o n o m y ~ a n d ~ A s t r o p h y s i c s, ~ L o s ~ A n g e l e s, ~ C a l i f o r n i a, ~}$ \\ United States
}


${ }^{a b}$ Caltech, Division of Physics, Mathematics and Astronomy, Pasadena, California, United States

\begin{abstract}
The Origins Space Telescope (Origins) concept is designed to investigate the creation and dispersal of elements essential to life, the formation of planetary systems, and the transport of water to habitable worlds and the atmospheres of exoplanets around nearby K- and M-dwarfs to identify potentially habitable - and even inhabited—worlds. These science priorities are aligned with NASA's three major astrophysics science goals: How does the Universe work? How did we get here? and Are we alone? We briefly describe the science case that arose from the astronomical community and the science traceability matrix for Origins. The science traceability matrix prescribes the design of Origins and demonstrates that it will address the key science questions motivated by the science case. (C) The Authors. Published by SPIE under a Creative Commons Attribution 4.0 Unported License. Distribution or reproduction of this work in whole or in part requires full attribution of the original publication, including its DOI. [DOI: 10.1117/1.JATIS.7.1 .011012]
\end{abstract}

Keywords: infrared; cryogenic space telescope; spectroscopy; galaxy evolution; planet formation; biosignatures.

Paper 20075SS received Jun. 14, 2020; accepted for publication Jan. 12, 2021; published online Feb. 27, 2021.

\title{
1 Introduction
}

The Origins Space Telescope (Origins) traces our cosmic history, from the formation of the first galaxies and the rise of metals to the development of habitable worlds and present-day life. Origins does this through exquisite sensitivity to infrared radiation from ions, atoms, molecules, dust, water vapor, and ice, and observations of extrasolar planetary atmospheres, protoplanetary disks, and large-area extragalactic fields. Origins operates in the wavelength range 2.8 to $588 \mu \mathrm{m}$ and is $>1000$ times more sensitive than its predecessors due to its large $\left(5.9 \mathrm{~m} / 25 \mathrm{~m}^{2}\right)$, cold $(4.5 \mathrm{~K})$ telescope, and advanced instruments. ${ }^{1}$

The science case for Origins was derived from the astronomical community through an extensive three-year exercise led by the Origins science working groups on galaxy evolution and cosmology, the Milky Way and nearby galaxies, planetary system formation, and exoplanets. The science input was prioritized and organized into three science themes that align with NASA's Astrophysics three thematic questions (Table 1). Each science theme has three science objectives that are specific questions that Origins will address. These science objectives specify the science requirements for the Origins design that are captured in the science traceability matrix (STM; Fig. 1).

In this paper, we briefly describe the science case and traceability matrix for Origins. The full science case is described in the Origins Space Telescope Study report. ${ }^{2,3}$ The science case builds upon expected advances from, and limitations of, current and next-generation observatories, namely, James Webb Space Telescope (JWST), ${ }^{4}$ Nancy Grace Roman Space Telescope, ${ }^{5}$ Atacama Large Millimeter Array (ALMA), ${ }^{6}$ and Vera C. Rubin Observatory. ${ }^{7}$ Origins addresses the key science goals by achieving its nine scientific objectives in 2 years. Thus, Origins will enable astronomers in the 2030s to ask new questions not yet imagined and provide a farinfrared window complementary to planned, next-generation observatories, such as the Laser Interferometer Space Antenna (LISA), ${ }^{8}$ Advanced LIGO, ${ }^{9}$ Athena, ${ }^{10}$ the Square Kilometer Array, ${ }^{11}$ and ground-based Extermely Large Telescopes (ELTs). ${ }^{12}$

The remainder of this paper is organized as follows. Section 2 describes the extragalactic case, which was coled by Alexandra Pope and Lee Armus. Section 3 highlights the planetary system formation case, which was coled by Klaus Pontoppidan, Edwin Bergin, and Kate Su.

\footnotetext{
*Address all correspondence to Margaret Meixner, mmeixner@usra.edu

${ }^{\dagger}$ Membership of the full Origins Space Telescope Study team can be found in the final report @ https://asd.gsfc.nasa.gov/firs/docs/ OriginsVolume1MissionConceptStudyReport25Aug2020.pdf
} 
Table 1 Mission design scientific drivers for the Origins Space Telescope.

\begin{tabular}{|c|c|c|c|}
\hline NASA goal & How does the universe work? & How did we get here? & Are we alone? \\
\hline $\begin{array}{l}\text { Origins } \\
\text { science } \\
\text { goals }\end{array}$ & $\begin{array}{l}\text { How do galaxies form stars, } \\
\text { make metals, and grow their } \\
\text { central SMBHs from } \\
\text { reionization to today? }\end{array}$ & $\begin{array}{l}\text { How do the conditions for } \\
\text { habitability develop during } \\
\text { the process of planet } \\
\text { formation? }\end{array}$ & $\begin{array}{l}\text { Do planets orbiting M-dwarf } \\
\text { stars support life? }\end{array}$ \\
\hline $\begin{array}{l}\text { Origins } \\
\text { scientific } \\
\text { capabilities }\end{array}$ & $\begin{array}{l}\text { With spectroscopic maps of } \\
\text { wide extragalactic fields, } \\
\text { Origins will simultaneously } \\
\text { measure properties of } \\
\text { growing SMBHs and their } \\
\text { galaxy hosts across cosmic } \\
\text { time. }\end{array}$ & $\begin{array}{l}\text { With sensitive, high- } \\
\text { resolution far-IR } \\
\text { spectroscopy, Origins maps } \\
\text { the water trail from } \\
\text { protoplanetary disks to } \\
\text { habitable worlds. }\end{array}$ & $\begin{array}{l}\text { With precise mid-infrared } \\
\text { transmission and emission } \\
\text { spectra, Origins will assess } \\
\text { the habitability of nearby } \\
\text { exoplanets and search for } \\
\text { signs of life. }\end{array}$ \\
\hline $\begin{array}{l}\text { Origins } \\
\text { scientific } \\
\text { objectives }\end{array}$ & $\begin{array}{l}\text { (1) How does the relative } \\
\text { growth of stars and } \\
\text { SMBHs in galaxies } \\
\text { evolve with time? } \\
\text { (2) How do galaxies make } \\
\text { metals, dust, and organic } \\
\text { molecules? } \\
\text { (3) How do the relative } \\
\text { energetics from } \\
\text { supernovae and quasars } \\
\text { influence the interstellar } \\
\text { medium of galaxies? }\end{array}$ & $\begin{array}{l}\text { (1) What role does water } \\
\text { play in the formation and } \\
\text { evolution of habitable } \\
\text { planets? } \\
\text { (2) How and when do } \\
\text { planets form? } \\
\text { (3) How were water and life's } \\
\text { ingredients delivered to } \\
\text { Earth and to exoplanets? }\end{array}$ & $\begin{array}{l}\text { (1) What fraction of terrestrial } \\
\text { planets around M- and } \\
\text { K-dwarf stars has } \\
\text { tenuous, clear, or cloudy } \\
\text { atmospheres? } \\
\text { (2) What fraction of terrestrial } \\
\text { M-dwarf planets is } \\
\text { temperate? } \\
\text { (3) What types of temperate, } \\
\text { terrestrial, M-dwarf } \\
\text { planets support life? }\end{array}$ \\
\hline
\end{tabular}

Section 4 provides an overview of the exoplanet case, which was coled by Kevin Stevenson, Tiffany Kataria, and Jonathan Fortney. Section 5 discusses the full Origins STM defined by the three themes and one imaging polarization case identified by the Milky Way and nearby galaxy science working group, which was coled by Cara Battersby and Karin Sandstrom. Section 6 discusses the discovery space for Origins.

\section{Extragalactic Case}

Origins will address the following key question about galaxy evolution: How do galaxies form stars, make metals, and grow their central supermassive black holes (SMBHs), from the Epoch of Reionization to today?

Decades of observations have shown that galaxies condensed out of primordial gas, built up their stellar mass, heavy metals, and central supermassive black holes (SMBHs), and evolved into the systems we see today. Yet we still do not understand how this happened. There is a rich interplay between the drivers of galaxy evolution, which can only be understood through new observations in a currently inaccessible wavelength regime, the far-infrared (Fig. 2).

Far-infrared observations are required because galaxies are dusty. Dust is a byproduct of star formation and is essential to astrophysical processes, from planetesimal formation in protoplanetary disks, to radiation-driven galactic outflows. ${ }^{14}$ Dust also obscures star formation and SMBH growth since it efficiently absorbs ultraviolet and optical light, rendering the driving processes of galaxy evolution nearly invisible at these wavelengths. However, the dust re-emits this energy in the far-infrared, making optically dim galaxies brilliant infrared sources. Just as importantly, infrared and molecular emission lines, which directly trace star formation, black-hole growth, and metal abundances, can escape dusty galaxies, making the mid- and far-infrared the only bands where a direct, unbiased view of the galaxy and metal growth is possible. ${ }^{15}$ This lesson was reinforced by Spitzer ${ }^{16}$ and Herschel,${ }^{17}$ which provided our first census of dusty galaxies in the infrared during the peak epoch of star formation, when the Universe was only 3 Gyr old. ${ }^{18}$ With 1000 times the sensitivity, Origins gives us a clear view of galaxy and metal growth across cosmic time, through a deep-and-wide 


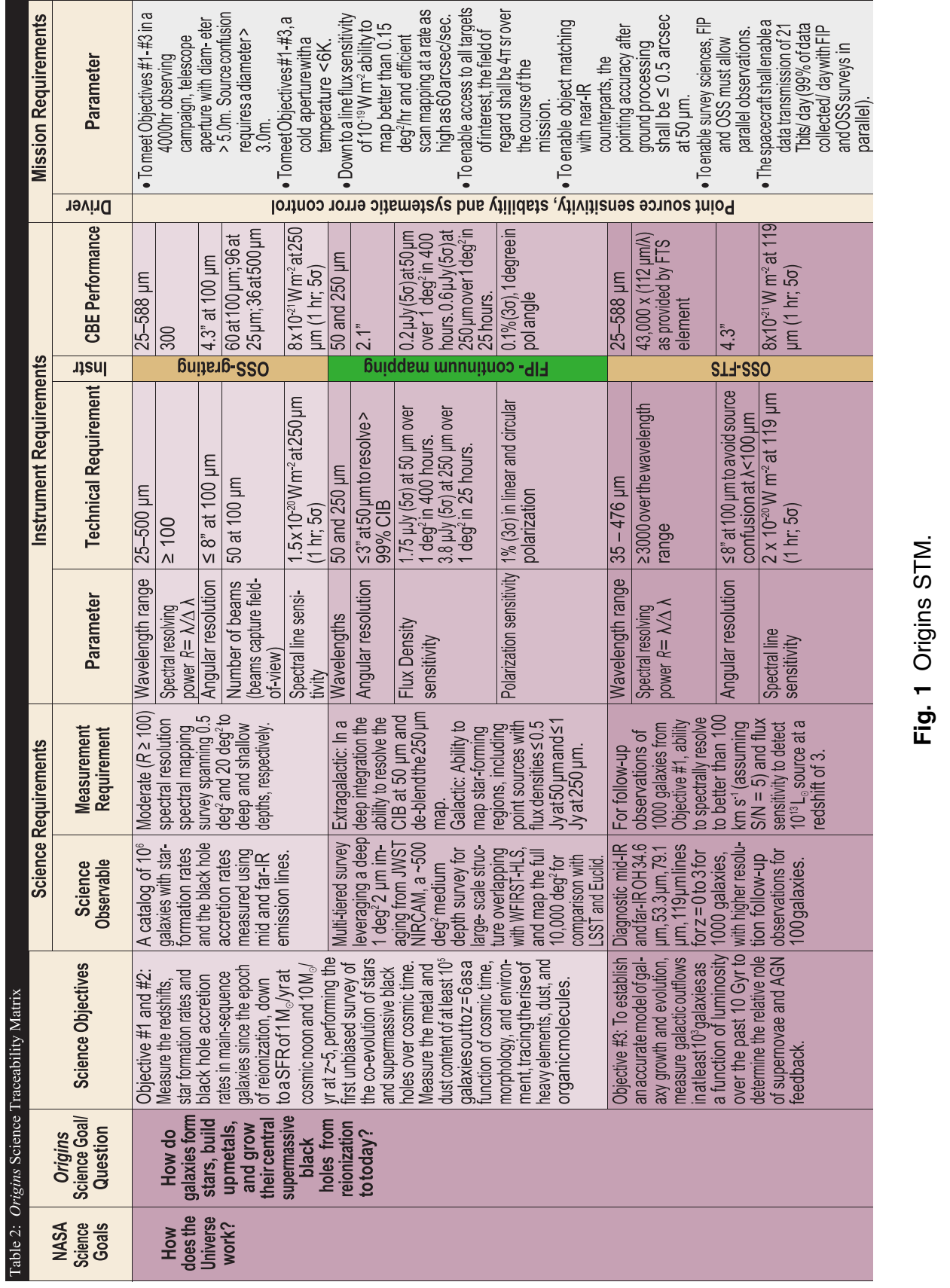




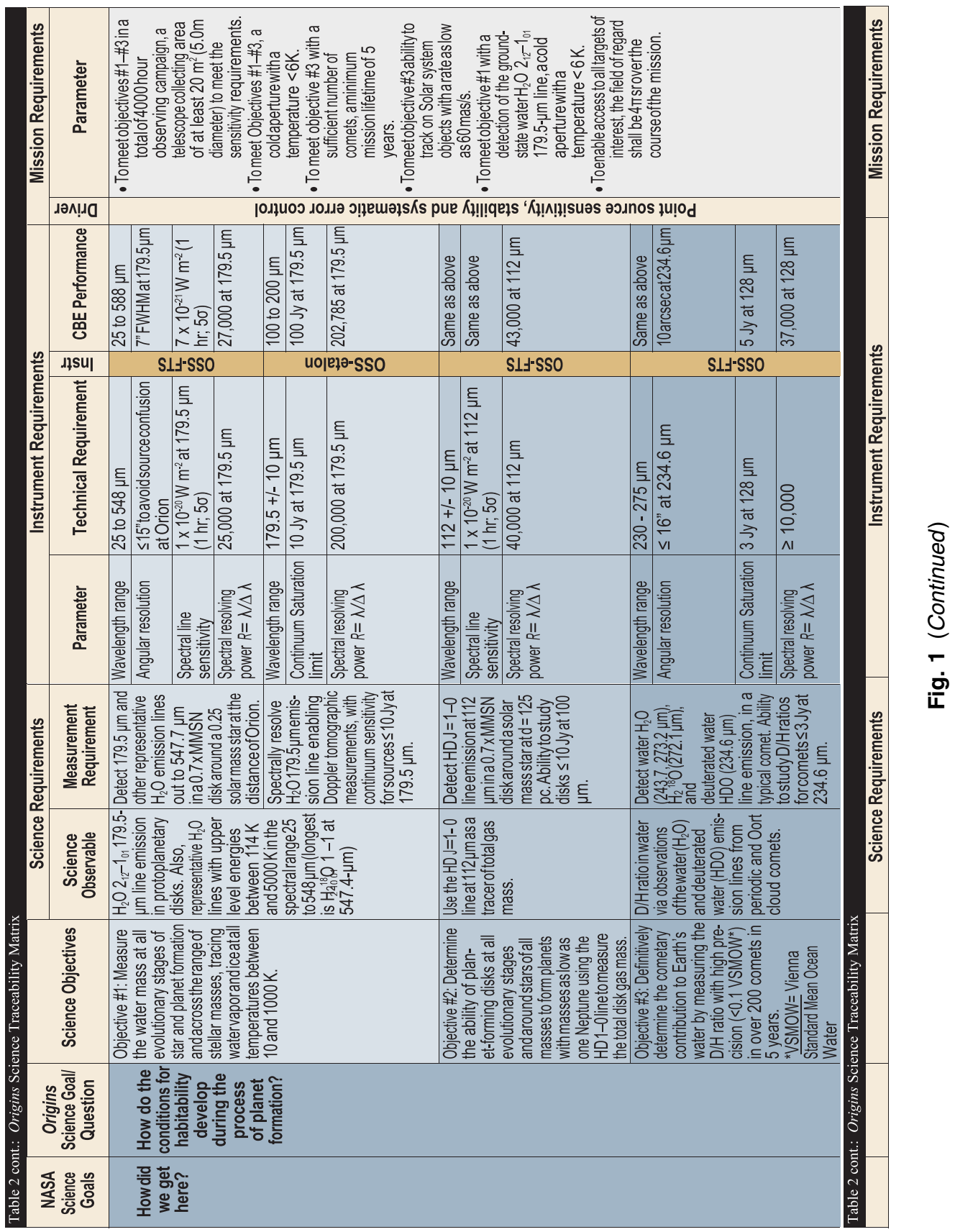




\begin{tabular}{|c|c|c|c|c|c|c|c|c|c|c|}
\hline 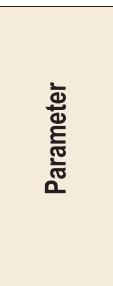 & \multicolumn{3}{|c|}{ 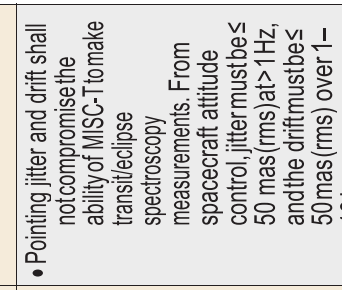 } & \multicolumn{6}{|c|}{ 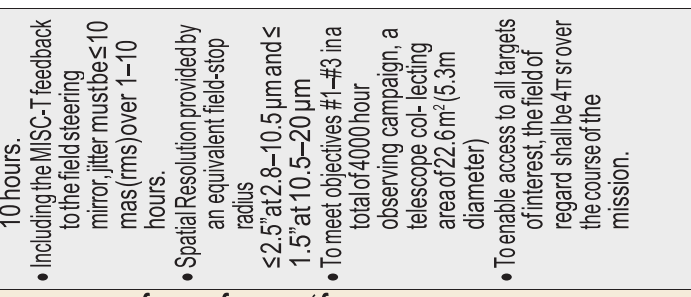 } & \\
\hline גәкा! & \multicolumn{10}{|c|}{ 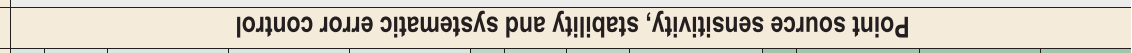 } \\
\hline 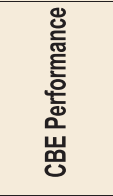 & 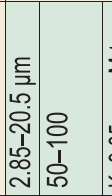 & 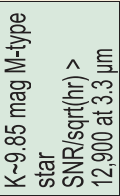 & 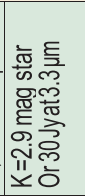 & 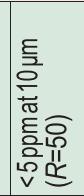 & 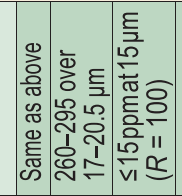 & 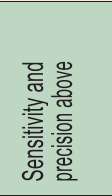 & & 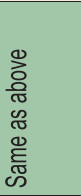 & 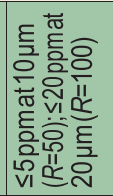 & 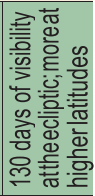 \\
\hline дsu| & \multicolumn{10}{|c|}{ 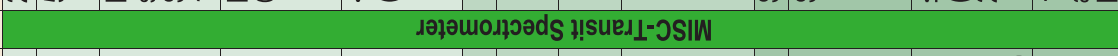 } \\
\hline 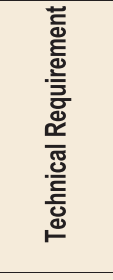 & 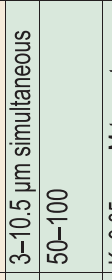 & 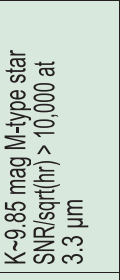 & 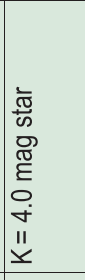 & 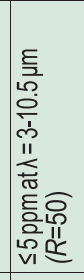 & 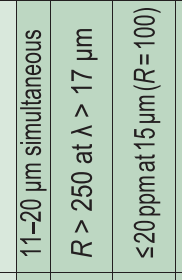 & 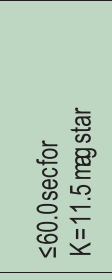 & & 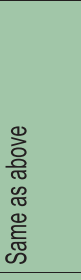 & 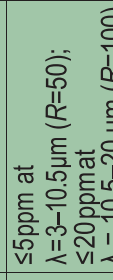 & 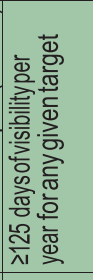 \\
\hline 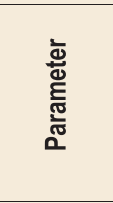 & 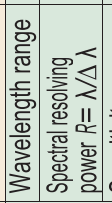 & 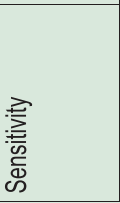 & 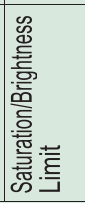 & 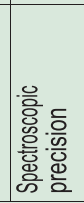 & 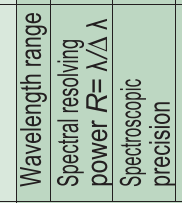 & 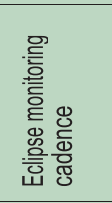 & & 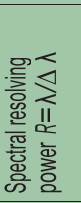 & 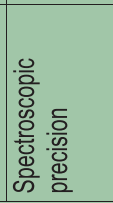 & 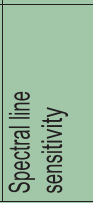 \\
\hline 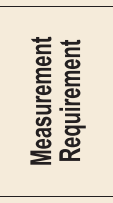 & \multicolumn{4}{|c|}{ 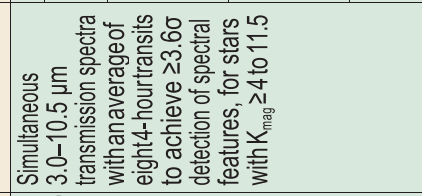 } & \multicolumn{2}{|c|}{ 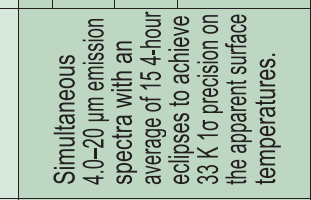 } & \multicolumn{4}{|c|}{ 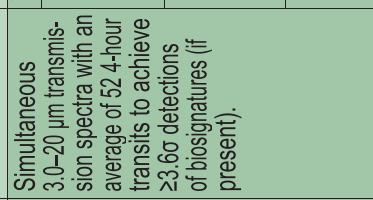 } \\
\hline 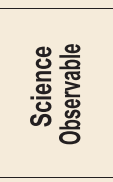 & \multicolumn{4}{|c|}{ 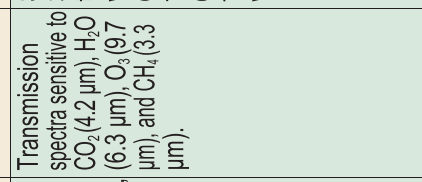 } & \multicolumn{3}{|c|}{ 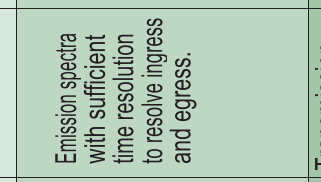 } & 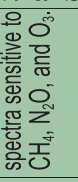 & & \\
\hline 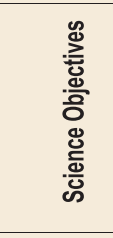 & \multicolumn{4}{|c|}{ 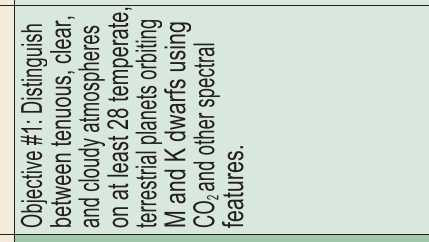 } & \multicolumn{6}{|c|}{ 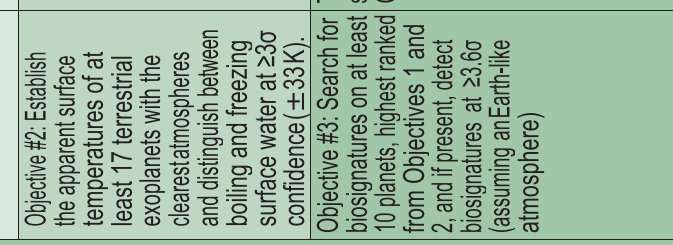 } \\
\hline 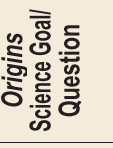 & \multicolumn{4}{|c|}{ 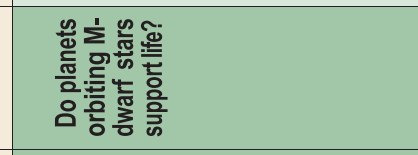 } & & & & & & \\
\hline 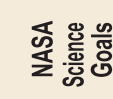 & \multicolumn{4}{|c|}{ 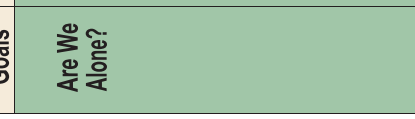 } & & & & & & \\
\hline
\end{tabular}




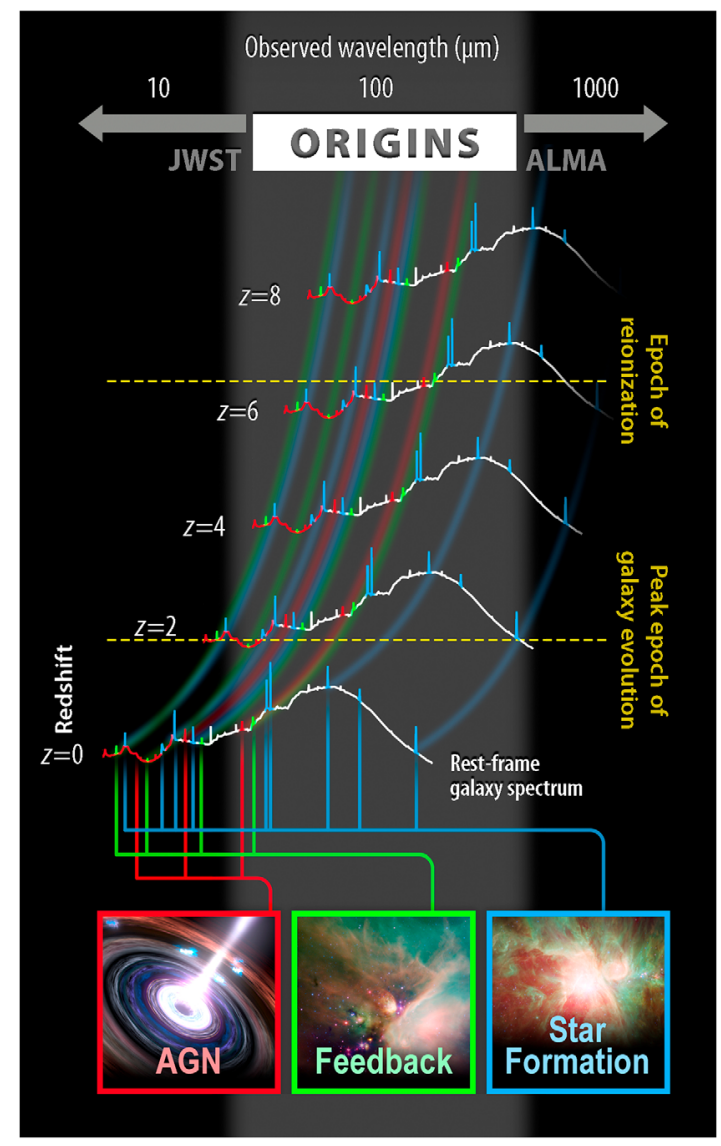

Fig. 2 Spectral reach of Origins over cosmic time showing a schematic representation of how the key spectral diagnostic features of active galactic nuclei (AGN, red), star formation (blue), and energetic feedback (green) move through the wide bandpass of the $\mathrm{OSS}^{13}$ with look-back time. Origins can measure all of these important processes over the entire history of galaxy evolution, filling in a key gap in wavelength and discovery space between JWST and ALMA.

spectroscopic survey in a wavelength regime inaccessible from the ground and that will remain unexplored by JWST. ${ }^{15}$

The over-arching extragalactic science goal is specifically addressed by three questions described below that correspond to science measurements in the traceability matrix.

\subsection{How Do the Stars and Supermassive Black Holes in Galaxies Evolve with Time?}

Origins uses atomic and molecular emission lines and emission from dust grains to measure the density, temperature, and ionization state of the gas where stars are forming and in galactic nuclei. ${ }^{15}$ These observations probe the physics of the interstellar medium, characterize the atomic and molecular gas that drives star-formation, measure the buildup of metals from dying stars, and track the growth of SMBHs and their influence, as they drive energetic outflows into the surrounding interstellar medium (Fig. 3).

\subsection{How Do Galaxies Make Metals, Dust, and Organic Molecules?}

Galaxies are the metal factories of the Universe, and Origins studies how heavy elements and dust were made and dispersed throughout the cosmic web over the past 12 billion years. ${ }^{19}$ Sensitive metallicity indicators in the infrared can be used to track the growth of heavy elements in even the densest optically obscured regions inside galaxies. 


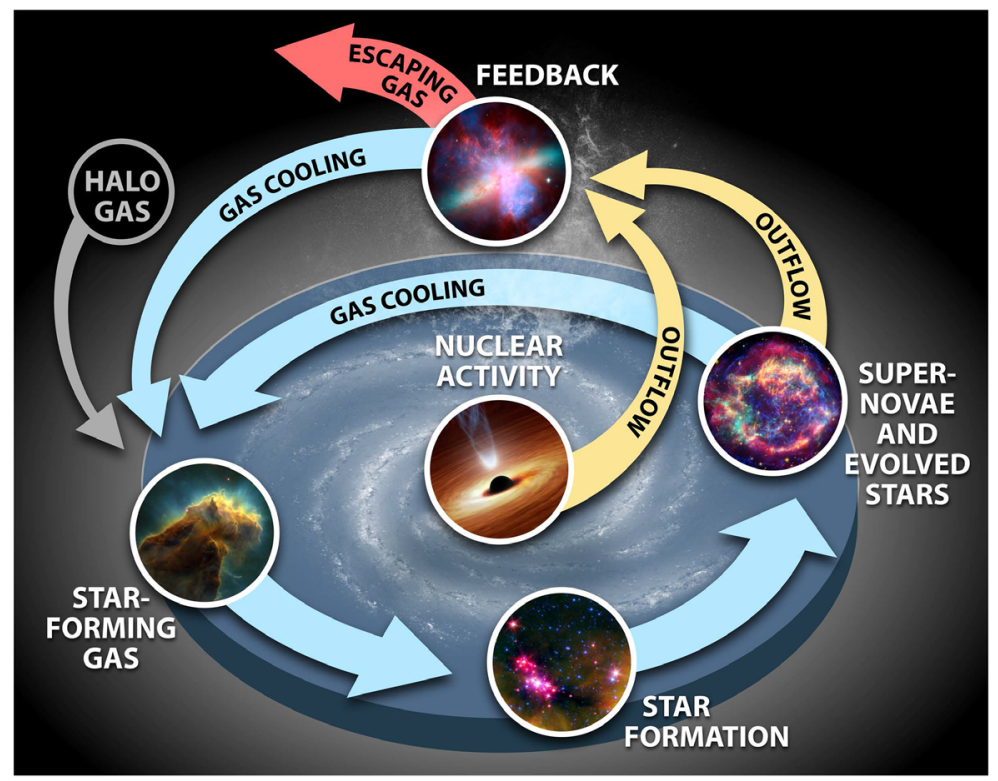

Fig. 3 Origins studies the baryon cycle in galactic ecosystems. Energetic processes that shape galaxies and the circumgalactic medium together define this ecosystem. Through its ability to measure the energetics and dynamics of the atomic and molecular gas and dust in and around galaxies, Origins can probe nearly all aspects of the galactic ecosystem: star formation and AGN growth; stellar death; AGN- and starburst-driven outflows; and gas cooling and accretion. These measurements provide a complete picture of the lifecycle of galaxies.

\subsection{How Do the Relative Energetics from Supernovae and Quasars Influence the Interstellar Medium of Galaxies?}

Galaxies are made of billions of stars, yet star formation is extremely inefficient on all scales, from single molecular clouds to galaxy clusters. The reason is thought to be "feedback" from star formation or black hole growth, because supernovae or quasar winds can disrupt star-forming gas. ${ }^{20}$ Origins studies the role of feedback processes in galaxies over a wide range of environments and redshifts by investigating the processes that drive powerful outflows and by surveying the demographics of galactic feedback.

\section{Planetary System Formation Case}

Origins will address the following key question about the formation and evolution of planetary systems (Fig. 4): How do the conditions for habitability develop during the process of planet formation?

Water is essential to all life on our planet. Water provides the liquid medium for life's chemistry and plays an essential biochemical role. However, we do not know how terrestrial planets get their liquid water. Rocky planets with liquid water exist in regions of the protoplanetary disk where water was not available during the planetary system formation because the star would have sublimated and photodissociated the water ice off the protoplanetary dust at this location. With its broad wavelength coverage, Origins can detect many water vapor lines that trace the entire range of temperatures found in protoplanetary disks, from the cold snowline to the hot steam line in disks (Fig. 5). ${ }^{21}$ Origins can survey all reservoirs of water in more than 1000 planetforming disks around stars of all masses, including the faint M-dwarfs that likely host most planets in the Galaxy.

Another puzzle in planetary formation is the role of hydrogen gas in protoplanetary disks. The disk's gas reservoir is essential to form gas giants. Moreover, the coupling of the gas to the dust may affect the formation and composition of planetesimals, which are the building blocks of 


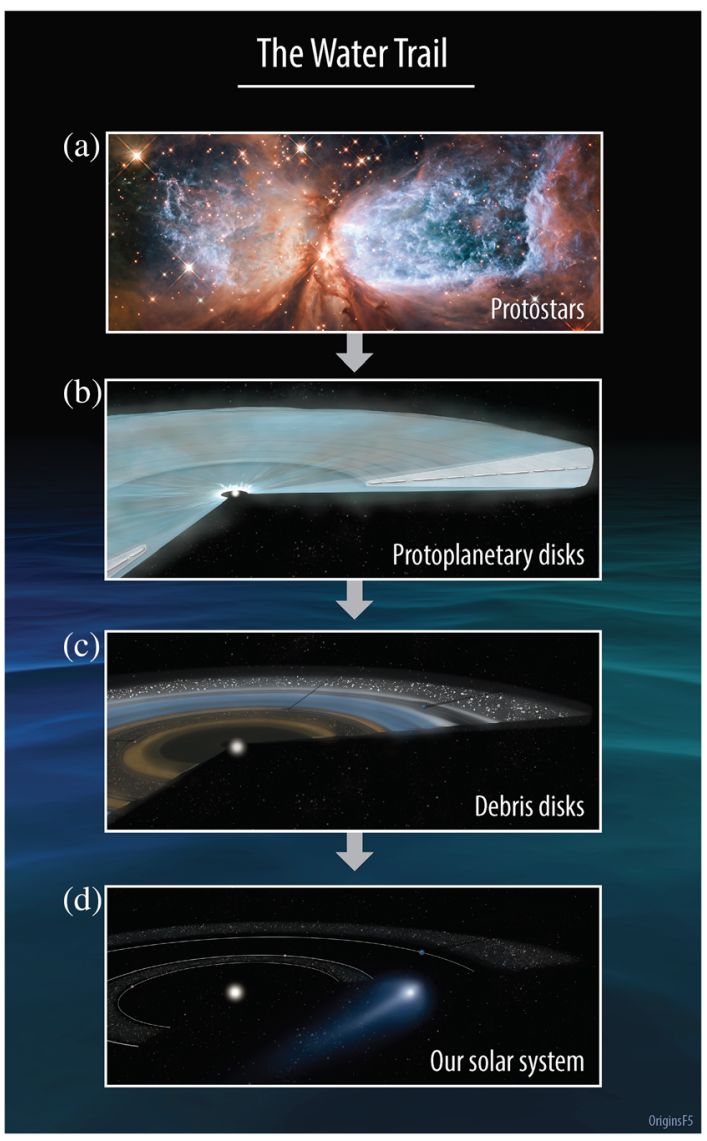

Fig. 4 Origins will trace water and gas during all phases of the formation of a planetary system. The trail begins in the "prestellar" phase explored by Herschel, where (a) a cloud of gas collapses into a still-forming star surrounded by a disk nearly the size of our solar system and (b) a collapsing envelope of material. Over time, (c) the envelope dissipates, leaving behind a young star and a disk with nascent planets, (d) eventually leaving behind a new planetary system. Origins will excel at probing the protoplanetary and later phases.

rocky planets. Despite decades of effort, the hydrogen gas mass of protoplanetary disks is essentially unknown because molecular hydrogen $\left(\mathrm{H}_{2}\right)$, being a symmetric molecule, requires significant energy to excite its rotational levels. Hence, molecular hydrogen is largely invisible at the low temperatures of planet-forming gas. Carbon monoxide (CO), which is the second most abundant molecule to $\mathrm{H}_{2}$, has been a traditional tracer for $\mathrm{H}_{2}$ in the interstellar medium. However, in protoplanetary disks $\mathrm{CO}$-estimated $\mathrm{H}_{2}$ masses are uncertain by factors of 10 to 100 in disks because the physical conditions in disks is very different than the insterstellar medium. Origins can observe the HD $112 \mu \mathrm{m}$ line, which provides a new and robust measure of disk gas mass. $^{22}$

The planetary system formation science goal is specifically addressed by three questions described below that correspond to science measurements in the traceability matrix.

\subsection{What Role Does Water Play in the Formation and Evolution of Habitable Planets?}

With its unprecedented sensitivity to weak emission from all forms of water (ice as well as gas), Origins deciphers the role of water throughout each phase of planetary system formation (Figs. 4 and 5). ${ }^{21}$ The Origins wavelength range includes warm water lines between 25 and $100 \mu \mathrm{m}$, and the ground-state lines at 179.5 and $538 \mu \mathrm{m}$. Origins observers will use these tracers to quantify the gas mass and the location of water in planet-forming disks. The model protoplanetary disk 


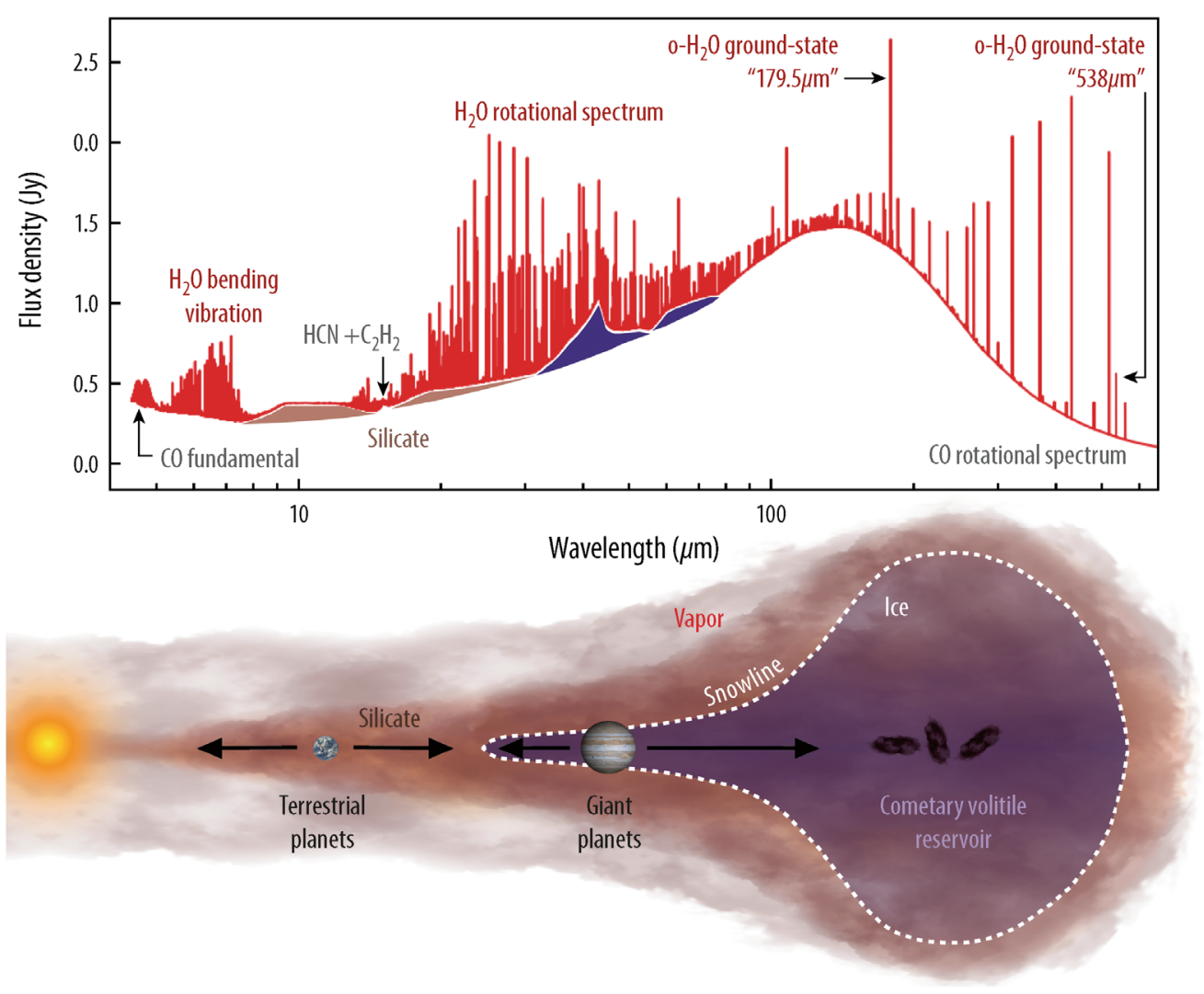

Fig. 5 Origins provides access to critical molecular tracers, including the HD $J=1-0$ line at $112 \mu \mathrm{m}$, and nearly the full $\mathrm{H}_{2} \mathrm{O}$ rotational spectrum. Also shown is a schematic of the different water regions in a planet-forming disk. The main regions include inner disk warm water vapor, midplane ice, and outer disk cold (photoevaporated) water vapor. Origins will probe the water and gas mass content throughout the disk.

spectrum in Fig. 5 is rendered at 4 to $660 \mu \mathrm{m}$ with a uniform $6 \mathrm{~km} \mathrm{~s}^{-1}$ spectral resolving power and for a disk distance of $125 \mathrm{pc}$. Previous spectroscopic observations of disks with Spitzer and Herschel were under-resolved, and therefore, did not show the dramatic line-to-continuum ratios to be revealed by Origins. JWST is only sensitive to warm, highly excited water lines near the star but not the cold-gas water lines that arise throughout the protoplanetary disk and serve as the best tracers of water during planetary system formation. Indeed, Origins accessibility to the cold water lines is very complementary to measurements made by JWST and ALMA, which are not as sensitive to water lines (Fig. 6).

\subsection{How and When Do Planets Form?}

Origins can uniquely use the HD $112 \mu$ m emission line, which is a powerful tool to measure the gas mass of protoplanetary disks to within a factor of 2 to $3 .^{22}$ This precision is one to two orders of magnitude better than alternative tracers and can distinguish between competing models of planet formation and set the timescale for gas-giant formation. Origins' gas-mass measurements have the potential to provide calibrations for all other observations of protoplanetary disks, including those made with ALMA using CO isotopes.

\subsection{How Were Water and Life's Ingredients Delivered to Earth and to Exoplanets?}

Earth likely formed within the snowline: the distance from a young star where water transitions from a gas to a solid. Thus, water was not a solid in the planetesimals where Earth formed. The 


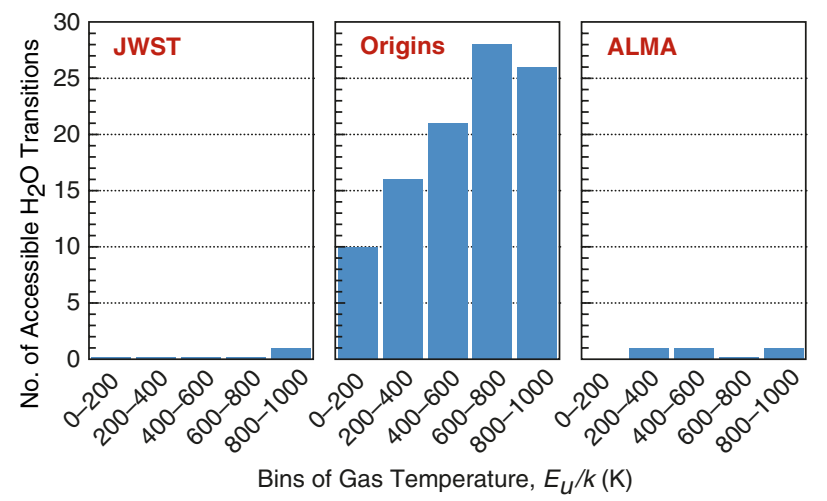

Fig. 6 Origins studies more than 100 transitions of the relevant water vapor lines, compared with one and three with JWST and ALMA, respectively. The plot shows the number of $\mathrm{H}_{2} \mathrm{O}$ transitions observable by JWST, Origins, and ALMA as a function of the gas temperature for energies above the ground state but less than $1000 \mathrm{~K}$ gas temperature. Gas temperatures less than $1000 \mathrm{~K}$ are the most relevant for studies of water throughout the protoplanetary disk and the formation of planetary systems. JWST probes only warm water lines close to the star. ALMA is limited by atmospheric absorption in its ability to observe water.

prevailing theory holds that water was delivered to the early Earth via impacts by bodies that formed beyond the snowline. The evidence for this comes from the relatively high deuterium content of Earth's oceans relative to the protosolar nebula, an excess that is locked when water is formed at a temperature of 10 to $20 \mathrm{~K} .{ }^{23}$ In our Solar System, comets and asteroids also carry this signature. Only a handful of comets have been measured to date and they show a range of deuterium abundances. With a larger sample of comets, Origins can finally establish the relative contributions of comets versus asteroids to the source of Earth's water.

\section{Exoplanets}

Origins will address the following key question about exoplanets: Do planets orbiting M-dwarf stars support life?

Humankind has long pondered the question, "Are we alone?" Our quest to search for life on planets around other stars relies on our ability to measure the chemical composition of their atmospheres and understand the data in the context of models for planet formation and evolution (Fig. 7). Using the techniques of transmission and emission spectroscopy, Origins will expand

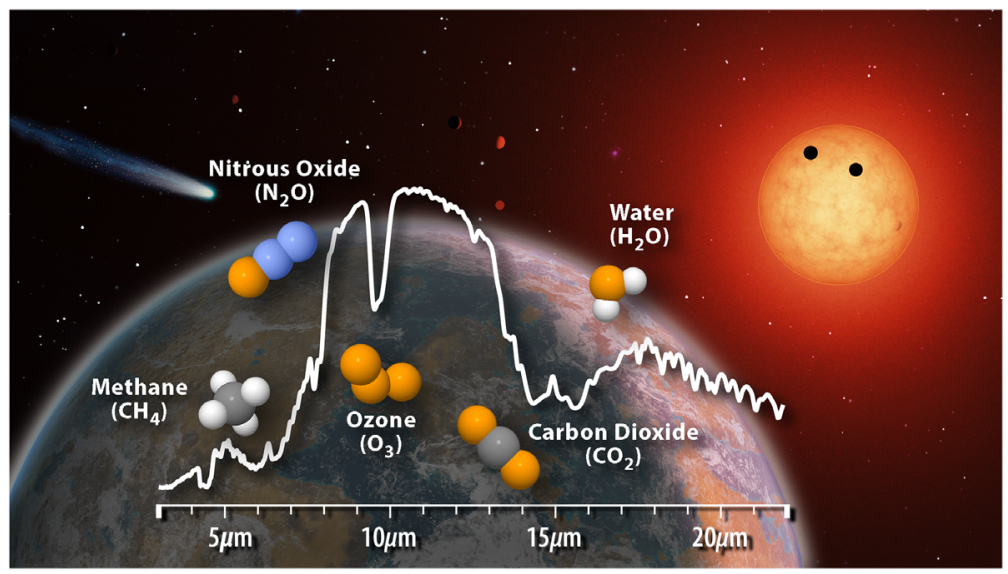

Fig. 7 Origins is designed to characterize already-discovered rocky planets that transit $\mathrm{M}$ dwarf stars and place critical constraints on their temperatures. By leveraging the midinfrared wavelengths offered by Origins, these atmospheres can be examined for gases that are the most important signatures of life. 

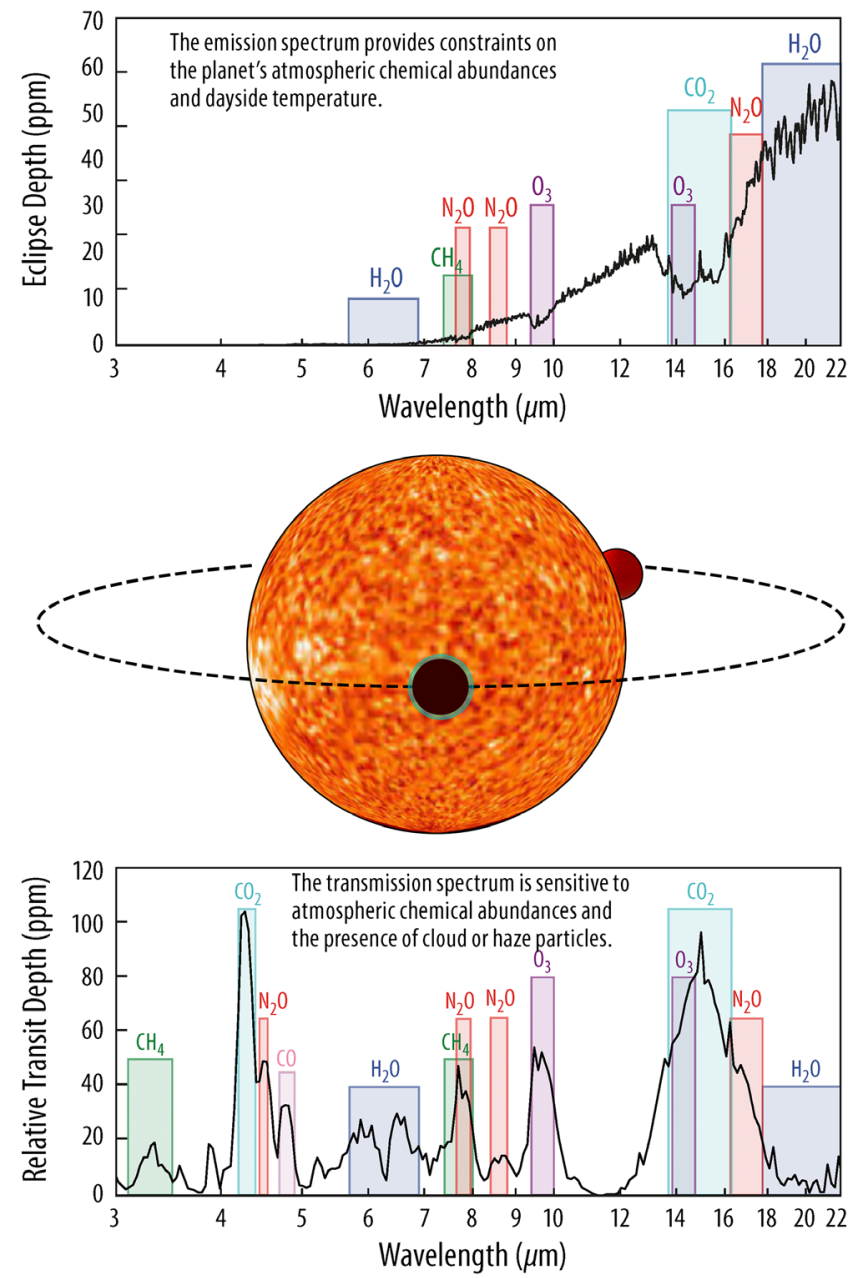

Fig. 8 The geometry and spectra for a typical transiting exoplanet. When a transiting planet passes in front of its host star, its apparent size changes as molecules absorb light at different wavelengths. When the planet passes behind the star, the planet's dayside thermal emission is measured, thus constraining a terrestrial planet's apparent surface temperature.

upon the legacy of Hubble and Spitzer - and soon JWST - with a midinfrared instrument specifically designed to characterize temperate, terrestrial exoplanets (Fig. 8). In its search for signs of life, Origins will employ a multitiered strategy, beginning with a sample of planets with well-determined masses and radii that are transiting nearby M dwarfs, the most abundant stars in the galaxy. ${ }^{24}$ With its broad, simultaneous wavelength coverage and unprecedented stability, Origins will be uniquely capable of detecting signs of life.

The exoplanet science goal is specifically addressed by three questions described below, which correspond to the three tiers of exoplanet measurements in the search for life in M-dwarfs. The sample size is largest for the first tier experiment/question and becomes smaller for the second tier and third tier experiments as only the most promising-for-life exoplanet candidates are promoted in the investigation. These three measurements involve transit spectroscopy (Fig. 8) and specify both instrument and observatory requirements in the STM.

\subsection{What Fraction of Terrestrial K- and M-Dwarf Planets Have Tenuous, Clear, or Cloudy Atmospheres?}

In the first tier of its exoplanet survey, Origins will obtain transmission spectra over 2.8 to $20 \mu \mathrm{m}$ for temperate, terrestrial planets spanning a broad range of planet sizes, equilibrium temperatures, and orbital distances, to distinguish between tenuous, clear, and cloudy atmospheres. 
An atmosphere that is large and uninhibited by clouds at these wavelengths will exhibit large spectral features such as $\mathrm{CO}_{2}$ in absorption. Because $\mathrm{CO}_{2}$ absorption features are so strong, this tier can include terrestrial planets orbiting stars from late-M to late- $\mathrm{K}$, giving Origins a broader perspective in the search for life than JWST.

\subsection{What Fraction of Terrestrial M-Dwarf Planets Is Temperate?}

For a subset of planets with the clearest atmospheres, Origins will measure their thermal emission to determine the temperature structure of their atmospheres. This is critical to assessing climate because it yields an understanding of how incoming stellar and outgoing thermal radiation dictate the heating and cooling of the atmosphere. Moreover, with this information the temperature at the surface of the planet can be estimated. Thus, Origins can then determine whether the planet could support liquid water near the surface.

\subsection{What Types of Temperate, Terrestrial M-Dwarf Planets Support Life?}

Origins will be the first observatory with the necessary spectroscopic precision to not only measure habitability indicators $\left(\mathrm{H}_{2} \mathrm{O}, \mathrm{CO}_{2}\right)$ but also crucial biosignatures $\left(\mathrm{O}_{3}\right.$ coupled with $\mathrm{N}_{2} \mathrm{O}$ or $\mathrm{CH}_{4}$ ), which are definitive fingerprints of life on habitable-zone planets. To address this third and most interesting question, Origins will obtain additional transit observations for the highestranked targets to search for and detect biosignatures with high confidence. The wavelength range afforded by Origins will provide access to multiple spectral lines for each molecular species. This will increase the detection significances and prevent potential degeneracies due to overlapping features, averting false positives. This framework robustly detects a variety of potentially habitable planet atmospheres, including those similar to the life-bearing Archaean Earth.

\section{Origins Science Traceability Matrix}

The Origins team took a holistic approach to the observatory by understanding not only the specific instrument and telescope needed to make a measurement but also how the observatory works to make the observation effectively and efficiently. Three instruments are specified to achieve the science goals of Origins: Origins Survey Spectrometer (OSS) ${ }^{13}$ Far-infrared Imager Polarimeter (FIP), ${ }^{25}$ and Mid-Infrared Spectrometer Camera Transit channel (MISC-T) ${ }^{26}$ Executing an observation requires understanding the choreography needed for the observatory ${ }^{1}$ and instruments to work together to make a measurement. Analysis of conceptual observations with specific operational modes has been conducted to identify key Mission Requirements, such as telescope diameter, attitude control requirements, and efficiency requirements.

The three Origins science themes and their science objectives are captured in the mission's STM (Fig. 1). The STM's six columns show the flow down from NASA science goals (column 1), to the Origins science theme question (column 2), to the prioritized science objectives (column 3), to science requirements (column 4), to the instrument requirements (column 5), and mission requirements (column 6). The STM rows are the three science themes. Science requirements (column 4) have two subcolumns: the science observable and its corresponding measurement requirement. The four instrument requirement (column 5) subcolumns show the parameter, technical requirement needed to achieve the science goal, the instrument, and the current best estimate (CBE) performance of that instrument based on the study. The science goals are traced from left to right (columns 1 to 5). The mission requirements column (6) presents overarching mission capabilities needed to support a science theme, such as telescope collecting area and observatory pointing stability, as these parameters also stem from the science measurement requirements.

To illustrate the requirements flow, let us follow the first science goal, column 2, "How do galaxies form stars, build up metals, and grow their central SMBHs from reionization to today?" This science goal is achieved with three science objectives noted in column 3 . The first objective is "measure the redshifts, star formation rates (SFR), and black hole accretion rates in mainsequence galaxies since the epoch of reionization down to an SFR of $1 \mathrm{M} \mathrm{yr}^{-1}$ at cosmic noon

J. Astron. Telesc. Instrum. Syst. $\quad$ 011012-13 Jan-Mar 2021 • Vol. 7(1) 
and $10 \mathrm{Myr}^{-1}$ at $z \sim 5$, performing the first unbiased survey of the coevolution of stars and SMBHs over cosmic time." To meet this objective, there are science requirements (column 4). The science observable (left subcolumn of column 4) is "A catalog of $10^{6}$ galaxies with starformation rates and black hole accretion rates measured using mid and far-IR emission lines." This science observable gives rise to a measurement requirement (right subcolumn of column 4) for "moderate $(R \geq 100)$ spectral resolution spectral mapping survey spanning $0.5 \mathrm{deg}^{2}$ and $20 \operatorname{deg}^{2}$ to deep and shallow depths, respectively." The instrument requirements (column 5) specify the technical requirement for each instrument parameter needed to meet the measurement requirement. For example, the parameter, wavelength range, has a technical requirement of 25 to $500 \mu \mathrm{m}$ for the OSS-grating mode of the OSS instrument. The concept OSS design has a CBE performance of 25 to $588 \mu \mathrm{m}$ for the wavelength range. The mission requirement (column 6) of telescope diameter is $>5.0 \mathrm{~m}$ if the extragalactic objectives\# 1 to 3 are to be completed in a 4000$\mathrm{h}$ observing campaign. However, the mission requirement of telescope diameter is $>3 \mathrm{~m}$ to resolve source confusion expected in spectral mapping surveys.

Several Origins programs (e.g., science objectives 1 and 2 for the extragalactic goal) require large-area sky surveys, with the consequence that Origins needs to accomplish large-area surveys efficiently. To support such surveys, the mission required an observatory survey mode that supports 60 arcsecond per second motion while observing. The optimal performance of far-infrared direct detectors is such that rapid motion of a source over the detectors is required, which necessitates on-sky scanning or motion of a field steering mirror. The instrument and observatory need stability to fulfill the mid-infrared spectroscopy of exoplanet goals. Science targets are located all over the sky, which means the field of regard over the year must cover the entire sky. Origins' requirement of $>80 \%$ observing efficiency guides attitude control system development and operational scenarios outlined in the science cases.

The science requirements specified in the STM set the minimum necessary to achieve the science goal. However, the Origins design is roughly $25 \%$ more capable than required by these science requirements (e.g., Fig. 9), leading to what is denoted as "science margin."

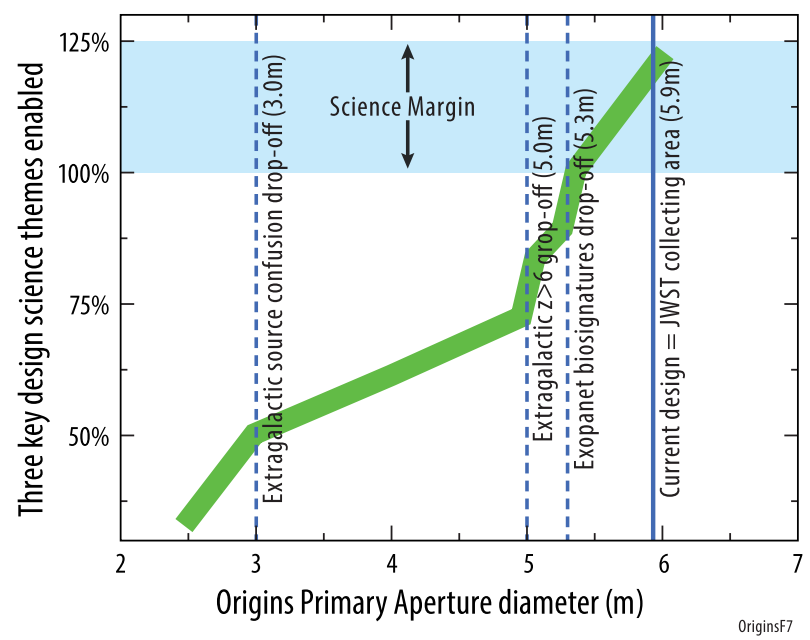

Fig. 9 Origins' key science program requires a cold telescope with a primary aperture diameter of $5.3 \mathrm{~m}$. This requirement comes primarily from the exoplanet science case to detect biosignatures in a 5-year mission, given that transit durations are fixed and sensitivity cannot be recovered with a longer single-epoch integration, unlike most other proposed Origins observations. The extragalactic study places an aperture size requirement of $>5 \mathrm{~m}$, based on the need to detect a statistically significant sample of galaxies at $z>6$, to study the formation mechanisms and physical properties of dust and metals during reionization. The minimum primary aperture diameter is $3 \mathrm{~m}$ to enable an effective extragalactic and Galactic science program, where source confusion does not compromise the telescope's ability to conduct spectroscopic studies of galaxies at $z=2$ to 3 and the sensitivity is not too poor to study water and gas in protoplanetary disks at the distances of Orion. 
The margin can be interpreted in two ways: margin captures the uncertainty in the model predictions or the margin implies that the scientific objectives can be reached with lower integration times than predicted in calculations for the report.

The baseline mission provides a good starting point upon Origins selection, with the flexibility to explore alternatives during an engineering phase A study. ${ }^{1}$ For example, Fig. 9 shows the fraction of each science theme that can be accomplished compared with the telescope diameter (collecting area). The baseline mission has a JWST collecting area (5.9-m diameter) that fits without deployments in the large launch vehicles under development. This size provides an estimated 25\% margin for Origins' science driver cases.

\section{Origins: Transformative Discovery Space}

Origins is designed for discovery. The three science goals that drive Origins' design can be achieved in $\sim 2$ years based on our observational program scenario assessments. Thus, there will be significant time to address other science topics. Indeed, Origins is a true community observatory, and its science program will be driven by science proposals selected through the usual peer-review process, as used for existing large NASA observatories.

The Origins-enabled scientific advances described above are extensions of known science questions. However, history has shown that order-of-magnitude leaps in sensitivity lead to discoveries of unanticipated phenomena. For example, the sensitivity of IRAS over balloon and airborne infrared telescopes led to the discovery of debris disks, protostars embedded within dark globules, Galactic infrared cirrus, and infrared-bright galaxies, none of which were expected at the time of launch. Likewise, no study anticipated that Spitzer would determine the stellar masses of $z>6$ galaxies and characterize the TRAPPIST-1 multiexoplanet system, the coldest known brown dwarfs, measure winds transporting energy in exoplanet atmospheres, and detect dust around white dwarfs produced by shredded asteroids.

Origins' sensitivity exceeds that of its predecessor missions by a factor of $1000 .{ }^{1} \mathrm{Jumps}$ of this magnitude are very rare in astronomy and have always revolutionized our understanding of the Universe in unforeseen ways. Moreover, the entire era of exoplanetary science has shown that Nature's imagination trumps our own, and Origins MISC-T's broad wavelength coverage and precise measurements are guaranteed to give us views into the new and unexpected. Thus, we anticipate a series of transformative discoveries by Origins that are impossible to imagine today.

\section{Acknowledgments}

The authors are grateful to the many institutions that sponsored and contributed to the successful Origins Space Telescope mission concept study. To enable the community to prepare for the 2020 Decadal Survey, NASA sponsored studies of four large mission concepts, of which Origins was one. We thank NASA for funding these studies. NASA's Goddard Space Flight Center (GSFC) contributed substantial additional labor support, which enabled us to explore options, make well-informed engineering decisions, and develop an executable mission concept. The Japanese space agency, JAXA, and a CNES-led European consortium actively participated in the study, with each contributing an instrument design and enabling their team members' travel to study team meetings and concurrent engineering sessions. Domestic study participants included many academic institutions, several NASA centers (Ames Research Center and Marshall Space Flight Center, as well as GSFC), the Caltech Jet Propulsion Laboratory, and industry (Ball Aerospace, Northrop-Grumman, Lockheed-Martin, and L3 Harris), as reflected in the authors' affiliations. Finally, the authors wish to express their deep gratitude to the hundreds of community members worldwide who contributed to the Origins mission concept study by sharing their thoughts on science priorities, reviewing the science case and engineering designs, developing graphics, formatting reports, taking notes, managing study resources, and making travel arrangements. It took a village. 


\section{References}

1. D. Leisawitz et al., "The Origins Space Telescope: trades and decisions leading to the baseline mission concept and future study topics," J. Astron. Telesc. Instrum. Syst. 7(1), (2021).

2. M. Meixner et al., "Origins Space Telescope mission concept study report," arXiv:1912. 06213 (2019).

3. M. Meixner et al., "Origins Space Telescope mission concept study report," 2019, https://asd .gsfc.nasa.gov/firs/docs/Origins Volume1MissionConceptStudyReport25Aug2020.pdf.

4. J. Gardner et al., "The James Webb space telescope," Sp. Sci. Rev. 123, 485 (2006).

5. "Nancy Grace Roman Space Telescope," https://roman.gsfc.nasa.gov (accessed 7 February 2021).

6. "Atacama Large Millimeter/submillimeter Array," https://www.almaobservatory.org/en/ home/ (accessed 7 February 2021).

7. "Vera C. Rubin Observatory," https://www.lsst.org (accessed 7 February 2021).

8. "Laser Interferometer Space Antenna," https://lisa.nasa.gov (accessed 7 February 2021).

9. The LIGO Collaboration, "Advanced LIGO," Classical and Quantum Gravity 32, 7 (2015).

10. "Advanced Telescope for High Energy Astrophysics," https://www.cosmos.esa.int/web/ athena (accessed 7 February 2021).

11. "Square Kilometre Array," https://www.skatelescope.org (accessed 7 February 2021).

12. "The Extremely Large Telescope," https://www.eso.org/public/teles-instr/elt/ (accessed 7 February 2021).

13. C. M. Bradford et al., "The Origins Survey Spectrometer (OSS): revealing the hearts of distant galaxies and forming planetary systems with ultrasensitive far-IR spectroscopy," JATIS, this volume (2020).

14. M. Melendez et al., "Exploring the dust content of galactic winds with Herschel. I. NGC 4631," Astrophys. J. 804, 46 (2015).

15. A. Pope et al., "Simultaneous measurements of star formation and supermassive black hole growth in galaxies," Bull. Am. Astron. Soc. 51(3), 330 (2019).

16. M. W. Werner et al., "The Spitzer space telescope mission," Astrophys. J. Suppl. Ser. 154, 1-9 (2004).

17. G. L. Pilbratt et al, "Herschel space observatory. An ESA facility for far-infrared and submillimetre astronomy," Astron. Astrophys. 518, L1-L6 (2010).

18. C. Casey et al., "Taking census of massive, star-forming galaxies formed $<1 \mathrm{Gyr}$ after the big bang," Bull. Am. Astron. Soc. 51(3), 212 (2019).

19. D. Burgarella et al., "Measuring the metallicity of low-mass, low-metallicity galaxies in the early universe and the galactic habitability," Astro2020: Decadal Survey on Astronomy and Astrophysics, science white papers, no. 213 (2019).

20. A. Bolatto et al., "Cold gas outflows, feedback, and the shaping of galaxies," Bull. Am. Astron. Soc. 51(3), 71 (2019).

21. K. Pontoppidan et al., "The trail of water and the delivery of volatiles to habitable planets," Bull. Am. Astron. Soc. 51(3), 229 (2019).

22. E. Bergin et al., "The disk gas mass and the far-IR revolution," Bull. Am. Astron. Soc. 51(3), 222 (2019).

23. D. Lis et al., "D/H ratio in water and the origin of earth's oceans," Bull. Am. Astron. Soc. 51(3), 111 (2019).

24. T. Kataria et al., "The mid-infrared search for biosignatures on temperate M-dwarf planets," Astro2020: Decadal Survey on Astronomy and Astrophysics, science white papers, no. 462 (2019).

25. J. Staguhn et al., "The Origins far-infrared imager and polarimeter (FIP)," J. Astron. Telesc. Instrum. Syst. 7(1), (2021).

26. A. Sakon et al., "The Origins mid-infrared spectrometer camera (MISC) baseline and upscope," J. Astron. Telesc. Instrum. Syst. 7(1), (2021).

Margaret Meixner is director of the SOFIA Science Mission Operations center. She received her bachelor's degree in electrical engineering and math from the University of Maryland, College Park, in 1987. She received her PhD in astronomy the University of California, 
Berkeley, in 1993. Her research includes infrared instrumentation and dust evolution in galaxies. She is a member of the JWST/MIRI Science Team, community cochair of NASA's STDT for the Origins Space Telescope mission concept. She was a JWST project scientist and Distinguished Astronomer at STScI. She is a fellow of the AAAS.

Asantha Cooray is on the faculty at the University of California, Irvine. He is a community cochair of NASA's STDT for the Origins Space Telescope mission concept.

David T. Leisawitz is NASA study scientist for the Origins Space Telescope and is most interested scientifically in the development of habitable conditions during planet formation. He was PI on the Space Infrared Interferometric Telescope mission concept study and served as NASA Goddard study lead for the Submillimeter Probe of the Evolution of Cosmic Structure. He was mission scientist for the Wide-field Infrared Survey Explorer and deputy project scientist for the Cosmic Background Explorer.

Lee Armus is a senior staff scientist at IPAC, lead scientist at the Roman Space Telescope Science Support Center, and a scientific editor for the Astrophysical Journal. He was formerly the lead for the Spitzer Space Telescope Infrared Spectrograph Instrument Support Team at the Spitzer Science Center. His research is focused on a number of topics central to the Origins mission, including the evolution of galaxies and supermassive black holes, and the study of infrared luminous galaxies across the electromagnetic spectrum.

Cara Battersby is an assistant professor of physics at the University of Connecticut. She received her PhD from the University of Colorado at Boulder, then held Submillimeter Array and National Science Foundation Postdoctoral Fellowships at the Harvard Smithsonian Center for Astrophysics She specializes in star formation in our Galaxy's Center. Her research group combines observational surveys with numerical simulations to understand the complex interplay of physics involved in star formation in this extreme environment.

Dominic Benford is an astrophysicist at NASA Headquarters, where he is the program scientist for the Nancy Grace Roman Space Telescope and for the Astrophysics Research and Analysis program. Prior to this, he served as the deputy mission scientist for the Wide-field Infrared Survey Explorer and the deputy PI of the Destiny Joint Dark Energy Mission formulation. In his astrophysics career, he developed technologies for the Spitzer and Herschel observatories and has worked on ground-based, balloon-borne, airborne, and sounding rocket projects for a range of long wavelength astrophysics investigations ranging from planetary atmospheres to the cosmic microwave background.

Charles Matt Bradford received his bachelor's degree in physics from Stanford University in 1994 and his doctorate in astronomy and instrumentation at Cornell in 2001. He held a Millikan postdoctoral fellowship at Caltech from 2001-2003 and has been on the science staff at JPL since that time. He has led the development and fielding of new submillimeter and millimeter-wave instrumentation on mountain-top site, using the datasets to study interstellar medium conditions in galaxies near and far. His current projects include a balloon-borne far-IR spectrometer and onchip mm-wave spectrometer, a mm-wave line intensity mapping instrument. He is also invested in developing concepts and the necessary detector systems for cryogenic space far-IR astrophysics missions.

Denis Burgarella is an astronomer in the Laboratoire d'Astrophysique de Marseille, AixMarseille University France. His preferred topic is the formation and evolution of galaxies and the quest to detect and understand the first stars and the first dust grains in the universe, from multi-wavelength data. He is at the origin of the SED modeling and fitting code CIGALE. $\mathrm{He}$ also is the present president of the IAU Division Cosmology and Galaxies.

Elvire De Beck is an assistant professor at Chalmers University of Technology and currently funded by the National Swedish Space Agency. She received her Msc and PhD degrees from KULeuven, Belgium. She was a fellow at the Max Planck Institute for Radio Astronomy in 
Bonn, Germany, after which she took positions as APEX staff astronomer and postdoctoral researcher at Chalmers University of Technology. Her research relates mainly to evolved stars, their outflows, and mass-loss mechanisms.

Kimberly Ennico-Smith has built infrared cameras, spectrometers, suborbital instruments, and lunar payloads. She has tested detectors at particle accelerators. She has served as SOFIA project scientist and New Horizons deputy project scientist. She has been an STDT member for Origins Space Telescope, authored 120+ peer-reviewed papers and delivered 50+ invited technical talks and 70+ public presentations. Asteroid 154587 Ennico is named for her. She is currently the VIPER lunar rover deputy project scientist.

Maryvonne Gerin, senior scientist at CNRS France, has extensive experience in studies of the diffuse and dense interstellar medium, with special emphasis on the chemical properties of the matter using ground based or spaceborne radio telescopes. She led the PRISMAS Herschel key program targeting hydrides in the diffuse interstellar medium. She is now the co-PI of the IRAM ORION-B large program.

Frank P. Helmich is head of the Astrophysics Program at SRON Netherlands Institute for Space Research. He obtained his PhD at Leiden Observatory. He moved to SRON, where he became the principal investigator for HIFI on the Herschel Space Observatory in 2007. He has been interim executive director for the Dutch research school for astronomy NOVA. He is alternate for the Netherlands delegation to the Science Program Committee of the European Space Agency.

Eric E. Mamajek is a deputy program chief scientist of the NASA Exoplanet Exploration Program, officed at the Jet Propulsion Laboratory. His research interest is the formation and evolution of exoplanetary systems and their host stars.

Gary J. Melnick is a senior astronomer at the Harvard-Smithsonian Center for Astrophysics specializing in infrared/submillimeter spectroscopy and astrochemistry. For more than 45 years, he has conducted airborne, balloon-borne, and space-based observations focused on the main coolants of interstellar clouds and understanding the formation, abundance, and distribution of interstellar water. He was PI of the SWAS Explorer mission, Co-I on the Herschel mission, and science lead for the Ices Investigation on the upcoming SPHEREx mission.

Stefanie N. Milam is an astrochemist with expertise in observations and spectroscopy at millimeter and submillimeter wavelengths and coordinating ground-based campaigns of cometary apparitions at multiple wavelengths. She also represents the planetary science community on a number of astrophysics missions and concepts including JWST, Roman Space Telescope, and the Origins Science and Technology Development Team.

Alexandra Pope (PhD University of British Columbia) is an associate professor of astronomy at the University of Massachusetts Amherst. She is an observational astronomer who specializes in infrared, (sub)millimeter, and radio observations of dusty galaxies at high redshift.

Thomas L. Roellig has worked in the NASA civil service as an astrophysicist at the NASA Ames Research Center since 1980 and is currently the chief of the astrophysics branch at Ames. His scientific research interests have spanned a wide range of infrared astronomy and astronomical instrumentation development. He has conducted research and published papers in infrared instrument development, solar science, solar system science, star formation, interstellar medium, and brown dwarf astronomy.

Douglas Scott (BSC Edinburgh, PhD Cambridge) is a professor of physics and astronomy at the University of British Columbia. He has coauthored more than 600 papers on a wide range of topics in cosmology, specializing in studies of the cosmic microwave background and submillimeter galaxies in the high-redshift Universe.

Kevin B. Stevenson is a staff astronomer at Johns Hopkins Applied Physics Laboratory and adjunct assistant professor in Johns Hopkins University's Department of Earth and Planetary 
Sciences. He is interested in characterizing the architectures and atmospheres of extrasolar planets to better understand their nature and origin; developing exoplanet mission concepts to measure the composition and chemical properties of nearby potentially-habitable worlds; and building software packages and tools to enable exoplanet observations and atmospheric characterization.

Martina C. Wiedner is a CNRS research scientist at Paris Observatory. She received her undergraduate degree from Karlsruhe University and her PhD from Cambridge University. She held an SMA postdoc at the Harvard-Smithsonian Center for Astrophysics and a post at the University of Cologne. She built and designed several heterodyne receivers, including the 1.4 THz CONDOR receiver and $183 \mathrm{GHz}$ water vapor monitors. She is interested in star formation and starburst galaxies.

Jonas Zmuidzinas is a professor of physics at Caltech. His research has centered on astrophysics at millimeter, submillimeter, and far-infrared wavelengths, including the development of instrumentation for ground-based, airborne, and space telescopes, and with a particular focus on the development of superconducting detectors. He currently serves as the director of the Caltech Optical Observatories.

Biographies of the other authors are not available. 